\title{
Fallbericht: Komplexe Posttraumatische Belastungsstörung
}

\author{
„Wenn ich Gefühle gezeigt habe, wurde mein Vater \\ nur noch wïtender und aggressiver"
}

\author{
Eine 51-jährige Patientin wurde seit dem frühen Kindesalter vom \\ Vater körperlich, sexuell und emotional missbraucht. Jahrelang hat \\ sie ihren Alltag bewältigt und funktioniert, bis eine transitorische \\ ischämische Attacke (TIA) die Diagnosestellung einer \\ Posttraumatischen Belastungsstörung nach sich zog.
}

\section{Symptome und Diagnose}

Behandlungsrahmen Die Patientin wird vorstellig im Rahmen der Aufnahme einer ambulanten Psychotherapie. Sie berichtet im Erstgespräch, vor 2 Jahren eine transitorische ischämische Attacke (TIA) aufgrund beruflicher Überforderung und Ängsten im Alltag gehabt zu haben. Im Rahmen des Krankenhausaufenthaltes sei dann erstmals eine komplexe Posttraumatische Belastungsstörung diagnostiziert worden.

Traumata Die Patientin wurde vom Vater seit dem 3. Lebensjahr sexuell, körperlich und emotional missbraucht. Die sexuellen Übergriffe endeten etwa mit dem 17. Lebensjahr. Anschließend habe er sie aber weiter massiv gedemütigt und kontrolliert. „Wenn ich Gefühle gezeigt habe, wurde mein Vater nur noch wütender und aggressiver.“ Um den Missbrauch aushalten zu können, dissoziierte die Patientin. Ferner berichtet sie, von 4 Männern in der S-Bahn vergewaltigt worden zu sein, als sie mit einer Freundin unterwegs war, sowie von 2 versuchten Vergewaltigungen im Alter von 13 und 17 Jahren.

PTBS-Symptomatik Die Patientin leide unter einer andauernden, hohen Anspannung und innerlicher Unruhe. Sie habe Einund Durchschlafprobleme sowie Konzentrationsprobleme. Ferner sei sie oft unsicher, schreckhaft und fühle sich ständig „auf der Flucht“. Etwa 2-mal wöchentlich habe sie aufdringliche Nachhallerinnerungen (Flashbacks) und dissoziiere. Aufgrund der hohen Ängstlichkeit vermeide die Patientin vielfältige Situationen, z.B. Fahrten mit öffentlichen Verkehrsmitteln, Spazieren in wenig belebten Gegenden, Prüfungen oder Situationen mit Körperkontakt, z. B. Massagen etc.

\section{Psychischer Befund}

- Die Patientin ist freundlich und zugewandt im Kontakt.

- Sie ist bewusstseinsklar und zu allen Qualitäten voll orientiert.

- Es findet sich kein Inhalt für eine wahnhafte Störung.

- Der formale Denkablauf ist grübelnd, ansonsten kohärent.

- Die Affektivität ist ängstlich-deprimiert, hoffnungslos, hilflos. Sie benennt Schuldgefühle und Minderwertigkeitsgefühle. Sie ist sehr angespannt, schreckhaft und innerlich unruhig.

- Suizidalität wird glaubhaft verneint.

- Die Konzentration ist vermindert, Aufmerksamkeit und Gedächtnis sind unbeeinträchtigt.

- Der Antrieb ist normal.

- Sie hat Einschlaf- und Durchschlafstörungen sowie Alpträume
- Sie hat Dissoziationen und Flashbacks.

- Sie hat Angst vor Autoritäten.

- Es findet sich kein Anhalt für eine Zwangsstörung.

- Die Drogen- und Alkoholanamnese erscheint unauffällig.

\section{Biografische/soziale Anamnese}

Familienanamnese Die Patientin wurde als 1 . Kind geboren. Ihr Vater (+25 Jahre) war sehr dominant, abwertend und stark kontrollierend. Ihre Mutter (+19 Jahre) war ängstlich, habe sich ihrem Ehemann untergeordnet und seine Übergriffe an ihrer Tochter ignoriert. Insgesamt sei die Patientin sehr isoliert aufgewachsen, da sie wenig nach draußen durfte. Die Großeltern mütterlicherseits waren wichtige Bezugspersonen, die jedoch weiter entfernt lebten. Mit ihrem Bruder (-3 Jahre) habe sie ein gutes Verhältnis gehabt; dieser sei aber anders erzogen worden.

Schulische Anamnese Die Patientin besuchte das Gymnasium und wechselte in der 8. Klasse auf die Realschule, da nach der Vergewaltigung in der S-Bahn ihre Leistungen eingebrochen seien. Mit 16 Jahren absolvierte sie den Realschulabschluss und wechselte dann erneut aufs Gymnasium. Mit 19 Jahren unterbrach sie die Schule, da ihre Eltern auswanderten und sie mitkommen musste. Mit 20 Jahren gelang ihr die Rückkehr nach Deutschland, sodass sie mit 21 Jahren ihr Abitur erlangte.

Berufliche Anamnese Schon mit 13 Jahren musste die Patientin nach der Schule in der Firma der Familie mitarbeiten. Ein Ausbildungsplatz wurde ihr nach 6 Monaten gekün- 
digt, da ihr Vater sie in Verruf gebracht habe. Das anschließende Studium sowie ein weiteres brach die Patientin nach 2 bzw. 3 Jahren wegen starker Prüfungsängste ab. Bis zur TIA arbeitete die Patientin als Altenpflegerin. Sie habe mehrmals Stellen gekündigt, um einen möglichen Konflikt zu verhindern. Aktuell ist eine Erwerbsminderungsrente beantragt.

\section{Beziehungsanamnese Die Patientin} musste mit 20 Jahren operiert werden, bevor sie mit ihrem ersten Freund schlafen konnte. In diesem Zusammenhang schilderte sie Schmerzen, fehlende Lubrikation und sexuelle Appentenz. Sie beschreibt, bezüglich des sexuellen Kontaktes „eine Nutzfläche“ gewesen zu sein. Mit 24 Jahren wurde sie schwanger und heiratete. Mit 26 Jahren wurde ihr 2. Kind geboren. Da sich ihr Ehemann finanziell von ihrem Vater abhängig machte, trennte sie sich nach 8 Jahren Ehe. Mit 33 Jahren war sie erneut schwanger und heiratete, trennte sich aber bereits nach einem Jahr, da ihr Mann sein Kind nicht anerkannte. Derzeit ist die Patientin in einer seit 7 Jahren bestehenden Partnerschaft. Es falle ihr schwer, ihrem Partner zu vertrauen, u. a. weil er sie einmal betrogen habe. Bei Problemen ziehe sie sich häufig zurück.

\section{Vorbehandlung}

- 1. stationäre Aufnahme für 3 Monate wegen Depressionen und Ängsten vor dem Hintergrund der Trennung von ihrem Mann mit 32 Jahren
- 2. stationäre Aufnahme für 3 Monate zur Behandlung der Posttraumatischen Belastungsstörung mit 49 Jahren

- 1. teilstationäre Behandlung der Posttraumatischen Belastungsstörung zur Verhinderung einer weiteren stationären Aufnahme mit 50 Jahren; anschließende ambulante Weiterbehandlung

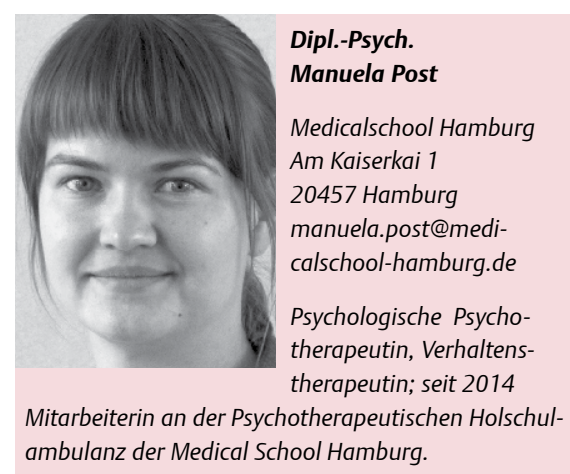

\section{Kommentare zum Fallbericht}

\section{Verhaltenstherapeutische Perspektive}

Störungsmodell Vor dem Hintergrund der Entwertung durch den Vater sowie der Verleumdung durch die Mutter (Ignorieren des Missbrauchs) und deren Rolle als Modell (ängstlich, untergeordnet, Frau als erduldendes, schwaches Geschlecht) sowie der damit einhergehenden fehlenden Befriedigung von Grundbedürfnissen (nach Grawe: Orientierung/Kontrolle, Lustgewinn/Unlustvermeidung, Bindung, Selbstwerterhöhung/-schutz) kam es durch die TIA zur Exazerbation der Symptomatik, welche Hilflosigkeits- und Ohnmachtsgefühle aktiviert hat. Die depressive Phase nach der Trennung vom ersten Ehemann (Ehe als Versuch, sich ein eigenes Leben aufzubauen) ist durch die finanzielle Abhängigkeit des Ehemanns vom Vater zu erklären, was zum einen weiteren Täterkontakt bedeutete, zum anderen einen Verbund gegen die Patientin und damit eine vergleichbare Situation wie früher (Mutter mit Vater) darstellte. Die Demütigung und Entwertung wurde somit vom Ehemann toleriert und die erlernte Hilflosigkeit der Patientin angestoßen. Die Konfliktvermeidung (angeregte Hilflosigkeitsschemata) entsteht vermutlich aus Sorge vor der der Omnipotenz des Interaktionspartners. Die Aufrechterhaltung kann durch den weiter bestehenden Täterkontakt, das Nichtvertrauen in der aktuellen Beziehung (Betrug durch den Partner), Vermeidung von Konflikten und angstauslösenden Situationen (u.a. öffentliche Verkehrsmittel, Körperkontakt) begründet werden. Insbesondere durch Letzteres kann kein Diskriminationslernen stattfinden.

\section{Behandlungsplan}

- Psychoedukation, Informationsvermittlung über Symptome, Ätiologie, Häufigkeit, Behandlungsmöglichkeiten; Erläuterung des Traumagedächnisses (klassisches Operantes Konditionieren)

- Erarbeiten eines Störungsmodells (Symptome, auslösende Situationen, Gefühle, Gedanken und Reaktionen)

- Erstellen einer Traumalandkarte (traumatische Ereignisse und Belastungen), explizite Analyse von Triggern

- „Stabilisierung“, Einüben von Selbstberuhigungsstrategien, Senkung der Anspannung (z.B. Tresorübung, Bildschirmtechnik, Achtsamkeitstraining, Atemtraining)

- Vorbereitung auf Traumaexposition, Durchführung durch Narration des traumatischen Ereignisses (Niederschreiben, IRRT, EMDR), Aufsuchen von vermiedenen Orten, Aktivitäten

- Kognitive Umstrukturierung: Überschätzung Gefahr, Schuld, Scham, Schwach, Beschmutzung

- Aufbau von Selbstwert und Selbstwirksamkeit, neue korrigierende Beziehungserfahrungen

- Validierung, Stärkung des „wohlwollenden Begleiters“, welcher die Möglichkeit zur Selbstfürsorge bietet

- Erwerb sozialer Kompetenzen: Abgrenzung, Nein-Sagen, Bedürfnisse und Gefühle wahrnehmen und mitteilen

Dr. rer. nat. Nadia Khan, Dipl.-Psych.

Psychologische Psychotherapeutin, Verhaltenstherapeutin. Seit 2014 Mitarbeiterin an der Psychotherapeutischen Holschulambulanz der Medical School Hamburg. 


\section{Kommentare zum Fallbericht}

\section{Systemischer Ansatz}

Veränderung versus Nichtveränderung Die Transitorische ischämische Attacke (TIA) scheint die Patientin „wie ein Blitz aus heiterem Himmel“ getroffen zu haben. Sie kann als Mitteilung ihres Körpers gedeutet werden, dass es so nicht mehr weitergehen kann. Die Patientin stellt, ausgelöst durch ihre langen Gewalterfahrungen, scheinbar seit ihrer Jugend unbewusst Situationen her (herausfordernde Partnerschaften, Arbeitsplatzwechsel bei Konflikten, sozialer Rückzug), in denen sie sich als ohnmächtig erlebt. Trotz des leidvollen Erlebens kann dies eine Form von Identität und Sicherheit schaffen, die die Patientin lange begleitet hat, jetzt aber nicht mehr reicht. Was hindert die Patientin daran, Veränderungen zuzulassen bzw. was trägt sie, aus gutem Grund, zur Nichtveränderung bei?

Beziehung zu den Angehörigen Alle Familienmitglieder sind vielschichtig belastet, auch wenn nur ein Mitglied der Familie einem Trauma ausgesetzt war. Gleichzeitig ist die Familie eine große Ressource in der Stabilisierung traumatisierter Menschen, deren Einbezug auch hier hilfreich sein kann. Zudem ist oft eine generationsübergreifende Perspektive erforderlich, um zu erkennen, welche traumatischen Ereignisse und welche damit verbundenen emotionalen Probleme von einer Generation auf die Nachfolgende übergeben wurden. Was wissen der Partner und die erwachsenen Kinder von der Patientin über ihre Lebensgeschichte? Wo können sie partiell in die ambulante Therapie einbezogen werden, um zur weiteren Stabilisierung der Patientin beizutragen? Welche Rolle haben die Eltern und der Bruder im weiteren Leben der Patientin gespielt, nachdem diese nach Deutschland zurückkehrte?

Ressourcenmobilisierung Gefühle von Ohnmacht und Hilfslosigkeit lassen die subjektive Überzeugung entstehen, keine Bewältigungsstrategien mehr zu haben. Die Ressourcen, über die die Patientin verfügt hat, sollen wieder bewusst wahrnehmbar gemacht und verankert werden. Wie hat es die Patientin über viele Jahre geschafft, den Alltag zu meistern, 3 Kinder zu bekommen und großzuziehen? In welchen Situationen hat sie sich schon etwas selbstbestimmt erlebt? Eine Hypothese von mir ist, dass sie sich bewusst gegen eine längere Therapie in der Zeit entschieden hat, als ihre Kinder noch klein waren, da sie befürchtete, für Kinder und Therapie gleichzeitig nicht stark genug zu sein.

Herstellung von Kontrolle/Sicherheit Die Biografie der Patientin zeigt deutlich, dass ihre Grundbedürfnisse nach Schutz, stabiler Bindung, Orientierung und Liebe durch ihre Eltern nicht erfüllt wurden. Ihr Vater hat sie über mehrere Jahre misshandelt, ihre Mutter hat sie nicht geschützt. Als Jugendliche erlebte die Patientin durch mehrfache Vergewaltigungen wieder einen Kontrollverlust, ebenso als junge Frau durch das erzwungene Auswandern mit den Eltern. Auch in ihrer beruflichen Situation scheint sie sich selten autark erlebt zu haben. Ein wesentliches Ziel der Behandlung wäre deshalb das Erlangen von innerer und äußerer Kontrolle.

\section{Jana von Majewsky}

Mag. Soziale Verhaltenswissenschaft, Erziehungswissenschaft, Soziologie, Systemische Familientherapeutin, Systemische Kinder- und Jugendlichentherapeutin, seit 2014 Mitarbeiterin an der Psychotherapeutischen Holschulambulanz der Medical School Hamburg.

\section{Tiefenpsychologische Überlegungen}

Beziehungs- und Objektrepräsentanzen Zum Verständnis der Patientin bzw. für die Indikationsstellung und Prognose einer psychodynamisch ausgerichteten Therapie wäre zunächst die Frage zu stellen, welche bedeutsamen zwischenmenschlichen Beziehungen aus Vergangenheit und Gegenwart das innere und interaktive Erleben der Patientin prägen. In Anbetracht eines missbrauchenden, demütigenden und kontrollierenden väterlichen Objekts, aber auch eines mütterlichen Objekts, das der Patientin weder Schutz noch Sicherheit bieten konnte, wäre zu explorieren, ob die Großeltern eine Basis für hinreichend stabile und integrierte Objektrepräsentanzen bieten konnten (was angesichts des über viele Jahre funktionierenden Kompensations- und Resilienzpotentials der Patientin zu vermuten wäre).

Dissoziative Symptomatik Die dissoziative Symptomatik scheint eine nützliche Abwehrfunktion übernommen zu haben, die es der Patientin ermöglicht hat, den Alltag zu meistern. Preis dafür ist die Notwendigkeit, Situationen, die an die abgespaltenen Inhalte rühren, im Alltag zu vermeiden. Dabei sind auch Formen der unbewussten inneren Vermeidungshaltung zu beachten, etwa das Selbsterleben als „Nutzfläche“ im sexuellen Kontakt: Hier hat die Patientin nicht nur schmerzliches, traumatisches Körpererleben komplett abgespalten, sondern scheint sich selbst überhaupt nicht mehr als lebendiges Wesen zu erleben. So kann sich das traumatische Szenario in den späteren Beziehungen scheinbar schmerzfrei reinszenieren.

Männerbeziehungen Bemerkenswert erscheint, dass es der Patientin gelang, sich aus der Beziehung mit dem Ehemann, der sich auch real in eine Verbindung mit dem Vater begeben hat, zu lösen. Dass dieser Befreiungsschlag die Abwehr vorübergehend destabilisierte, sodass eine stationäre Behandlung erforderlich wurde, ist kaum verwunderlich. Die Schilderung der auf die Ehe folgenden Beziehungen legt nahe, dass der Patientin eine innere Loslösung aus dem maligne-abhängigen Beziehungsmuster mit dem Vater weiterhin nicht möglich war. 
Mütterliches Muster Auch Überlegungen, warum die PTBS trotz der bereits bestehenden psychischen Störungen und erfolgten Behandlung erst auffallend spät als solche diagnostiziert wurde, können psychodynamisch bedeutsam sein: Die früheren Behandler scheinen das Muster der Mutter fortzusetzen, die psychischen und physischen Übergriffe werden ignoriert. Durch diese Aufrechterhaltung des mütterlichen Musters entkommt die Patientin dem schmerzlichen Erleben, von der Mutter damals schutzlos allein gelassen worden zu sein.

Fokus und Behandlungsplanung Zentral für eine tiefenpsychologisch fundierte Psychotherapie ist die Orientierung an einem zu Beginn zu definierenden Behandlungsfokus. Bei der Patientin scheint aktuell die Frage anzustehen, ob sie ihrem Partner vertrauen oder sich innerlich von ihm lösen kann. Dies lässt sich zugleich als zentrale Fragestellung bezogen auf ihre inneren Objekt- und Beziehungsrepräsentanzen verstehen. Die Arbeit an der traumatischen Beziehungsrepräsentanz und am Täterintrojekt, aber auch das Herausarbeiten der „guten“, vertrauenswürdigen Repräsentanzen und letztlich die Überwindung der Spaltung wären in der Therapie anzustreben.

Übertragungsaspekte $\mathrm{Zu}$ beachten ist, dass die therapeutische Ich-Spaltung im Kontext traumatischer Inhalte häufig beeinträchtigt ist. Der Therapeutin werden dann schnell Tätereigenschaften zugeschrieben, die von der Patientin nicht mehr von der Realität zu unterscheiden sind, sodass ein solches Erleben einer Retraumatisierung gleichkommt. Der Grundsatz der tiefenpsychologisch fundierten Therapie, nicht so sehr in der Übertragung, sondern vielmehr mit der Übertragung zu arbeiten, erscheint mit einer traumabezogenen Arbeitsweise somit sehr gut vereinbar. Im Idealfall wäre eine Vertrauensbeziehung mit einer positiven, nicht idealisierenden Übertragung als Basis für jede inhaltliche Arbeit zu Behandlungsbeginn zu etablieren und auch im Verlauf immer wieder neu zu erarbeiten.

\section{Dipl.-Psych. Verena Daues}

Psychologische Psychotherapeutin, Tiefenpsychologisch fundierte Psychotherapie. Seit 2014 Mitarbeiterin an der Psychotherapeutischen Hochschulambulanz der Medical School Hamburg.

\section{Psychoanalytische Perspektive}

Vorbemerkung Die Sachlichkeit, mit der die Patientin beschrieben wurde, hinterlässt beim ersten Lesen des Fallberichts eine seltsame Art von Unbekümmertheit; erst beim zweiten Lesen stellt sich ein schreckliches Grauen ein. Dieser Widerspruch mag einen Eindruck davon vermitteln, wie die Patientin innerlich mit den Katastrophen der Vergangenheit umgehen muss: Die Gefühle von vollständiger Ohnmacht und Ausgeliefertsein, der Schmerz und die Wut dürfen nicht ins Bewusstsein gelangen - sie wären zu überwältigend. Da nicht ausgeführt ist, wie es der Therapeutin im Erstgespräch mit der Patientin innerlich ergangen ist, bleiben für den Psychoanaly- tiker einige wichtige Fragen für das Verständnis der Patientin und ihrer inneren Welt offen: Wie hat sie sich im ersten persönlichen Kontakt präsentiert? Wie ihr Anliegen vorgetragen? Welche Gefühle, Fantasien und Impulse hat dies in der Untersucherin hervorgerufen?

Ausgangssituation Eine TIA zeigt sich in Form von Lähmungen, Sprach- und Wahrnehmungsstörungen. Das weckt Fantasien, dass die Patientin ihre innere Ohnmacht und ihren Wunsch nach Hilfe nur über körperliche Symptome zum Ausdruck bringen kann. Als aktueller Auslöser der später erwähnten PTBS-Symptome wird eine berufliche Überforderung benannt. Der jahrelange Missbrauch durch den Vater, später wiederholt in den Partnerschaften der Patientin, scheint ursächlich, wobei offen bleibt, ob die Patientin diesen Zusammenhang selbst herstellen kann. Die Angst vor weiteren Grenzüberschreitungen wird vor diesem Hintergrund psychodynamisch verstehbar.

Psychodynamik Als ältestes Kind einer ängstlichen, schwachen Mutter und eines dominanten, gewalttätigen Vaters war die Patientin früh schutzlos schlimmsten Grenzüberschreitungen ausgeliefert. Dies dürfte in ihr das Bild hinterlassen haben, dass es in Beziehungen keine Sicherheit gibt und dass ihre eigenen Wünsche und Bedürfnisse nichts zählen. Vermutlich gibt sich die Patientin unbewusst selbst die Schuld für die Grenzüberschreitungen des Vaters, was es ihr, zusätzlich zur Scham, erschwert, sich Hilfe zu holen. In späteren Beziehungen kommt es immer wieder zur Reinszenierung der Missbrauchserfahrungen. Die Sexualität der Patientin ist schwer belastet: Lustvolle Erfahrungen erscheinen unmöglich, und der ärztliche Eingriff zur Herstellung ihrer sexuellen „Funktionsfähigkeit“ erscheint wie ein weiterer Übergriff. Wut und Enttäuschung über die Grenzverletzungen sowie den mangelnden Schutz durch die Mutter werden abgespalten (Dissoziation) oder gegen das Selbst gerichtet (Depressionen). Eigene Bedürfnisse kann die Patientin nur indirekt befriedigen, z.B. über die Versorgung Anderer (Altenpflege). Die Überforderung im Job sowie ihre Schwierigkeiten, Konflikte offen auszutragen (Angst vor erneuten Übergriffen), schwächen die Abwehr immer mehr.

Indikation und Prognose Damit sich die Patientin in einem verlässlichen, sicheren Rahmen vertrauensvoll einlassen kann, wäre m. E. eine langjährige, höherfrequente Psychotherapie nötig. Wichtigstes Ziel sollte, neben einer Stärkung des Selbstvertrauens, die innere Distanzierung vom Täter-Introjekt sein. Diese kann nur stattfinden, wenn die Patientin sich mit der Person eines verlässlichen, zugewandten und v. a. abstinenten Analytikers identifizieren kann, um so zu mehr innerer Sicherheit zu gelangen. Gelingt dies, würde ich von einer ausreichend günstigen Prognose ausgehen.

\section{Dipl.-Psych. Thomas Völker}

i. A. zum Psychologischen Psychotherapeuten und Psychoanalytiker, 9 Jahre im Universitätsklinikum Hamburg-Eppendorf; seit April 2014 Mitarbeiter in der Psychotherapeutischen Hochschulambulanz der Medical School Hamburg.

Beitrag online zu finden unter http://dx.doi.org/ 